\title{
Review articles: purpose, process, and structure
}

\author{
Robert W. Palmatier ${ }^{1} \cdot$ Mark B. Houston $^{2} \cdot$ John Hulland $^{3}$
}

Published online: 2 October 2017

(C) Academy of Marketing Science 2017

Many research disciplines feature high-impact journals that are dedicated outlets for review papers (or review-conceptual combinations) (e.g., Academy of Management Review, Psychology Bulletin, Medicinal Research Reviews). The rationale for such outlets is the premise that research integration and synthesis provides an important, and possibly even a required, step in the scientific process. Review papers tend to include both quantitative (i.e., meta-analytic, systematic reviews) and narrative or more qualitative components; together, they provide platforms for new conceptual frameworks, reveal inconsistencies in the extant body of research, synthesize diverse results, and generally give other scholars a "state-of-the-art" snapshot of a domain, often written by topic experts (Bem 1995). Many premier marketing journals publish meta-analytic review papers too, though authors often must overcome reviewers' concerns that their contributions are limited due to the absence of "new data." Furthermore, relatively few non-meta-analysis review papers appear in marketing journals, probably due to researchers' perceptions that such papers have limited publication opportunities or their beliefs that the field lacks a research tradition or

Robert W. Palmatier

Palmatrw@uw.edu

Mark B. Houston

m.b.houston@tcu.edu

John Hulland

jhulland@uga.edu

1 Foster School of Business, University of Washington, Box: 353226, Seattle, WA 98195-3226, USA

2 Neeley School of Business, Texas Christian University, Fort Worth, TX, USA

3 Terry College of Business, University of Georgia, Athens, GA, USA "respect" for such papers. In many cases, an editor must provide strong support to help such review papers navigate the review process. Yet, once published, such papers tend to be widely cited, suggesting that members of the field find them useful (see Bettencourt and Houston 2001).

In this editorial, we seek to address three topics relevant to review papers. First, we outline a case for their importance to the scientific process, by describing the purpose of review papers. Second, we detail the review paper editorial initiative conducted over the past two years by the Journal of the Academy of Marketing Science (JAMS), focused on increasing the prevalence of review papers. Third, we describe a process and structure for systematic (i.e., non-meta-analytic) review papers, referring to Grewal et al. (2018) insights into parallel meta-analytic (effects estimation) review papers. (For some strong recent examples of marketing-related meta-analyses, see Knoll and Matthes 2017; Verma et al. 2016).

\section{Purpose of review papers}

In their most general form, review papers "are critical evaluations of material that has already been published," some that include quantitative effects estimation (i.e., meta-analyses) and some that do not (i.e., systematic reviews) (Bem 1995, p. 172). They carefully identify and synthesize relevant literature to evaluate a specific research question, substantive domain, theoretical approach, or methodology and thereby provide readers with a state-of-the-art understanding of the research topic. Many of these benefits are highlighted in Hanssens' (2018) paper titled "The Value of Empirical Generalizations in Marketing," published in this same issue of JAMS.

The purpose of and contributions associated with review papers can vary depending on their specific type and research question, but in general, they aim to 
- Resolve definitional ambiguities and outline the scope of the topic.

- Provide an integrated, synthesized overview of the current state of knowledge.

- Identify inconsistencies in prior results and potential explanations (e.g., moderators, mediators, measures, approaches).

- Evaluate existing methodological approaches and unique insights.

- Develop conceptual frameworks to reconcile and extend past research.

- Describe research insights, existing gaps, and future research directions.

Not every review paper can offer all of these benefits, but this list represents their key contributions. To provide a sufficient contribution, a review paper needs to achieve three key standards. First, the research domain needs to be well suited for a review paper, such that a sufficient body of past research exists to make the integration and synthesis valuable-especially if extant research reveals theoretical inconsistences or heterogeneity in its effects. Second, the review paper must be well executed, with an appropriate literature collection and analysis techniques, sufficient breadth and depth of literature coverage, and a compelling writing style. Third, the manuscript must offer significant new insights based on its systematic comparison of multiple studies, rather than simply a "book report" that describes past research. This third, most critical standard is often the most difficult, especially for authors who have not "lived" with the research domain for many years, because achieving it requires drawing some nonobvious connections and insights from multiple studies and their many different aspects (e.g., context, method, measures). Typically, after the "review" portion of the paper has been completed, the authors must spend many more months identifying the connections to uncover incremental insights, each of which takes time to detail and explicate.

The increasing methodological rigor and technical sophistication of many marketing studies also means that they often focus on smaller problems with fewer constructs. By synthesizing these piecemeal findings, reconciling conflicting evidence, and drawing a "big picture," meta-analyses and systematic review papers become indispensable to our comprehensive understanding of a phenomenon, among both academic and practitioner communities. Thus, good review papers provide a solid platform for future research, in the reviewed domain but also in other areas, in that researchers can use a good review paper to learn about and extend key insights to new areas.

This domain extension, outside of the core area being reviewed, is one of the key benefits of review papers that often gets overlooked. Yet it also is becoming ever more important with the expanding breadth of marketing (e.g., econometric modeling, finance, strategic management, applied psychology, sociology) and the increasing velocity in the accumulation of marketing knowledge (e.g., digital marketing, social media, big data). Against this backdrop, systematic review papers and meta-analyses help academics and interested managers keep track of research findings that fall outside their main area of specialization.

\section{$J A M S$ ' review paper editorial initiative}

With a strong belief in the importance of review papers, the editorial team of JAMS has purposely sought out leading scholars to provide substantive review papers, both metaanalysis and systematic, for publication in JAMS. Many of the scholars approached have voiced concerns about the risk of such endeavors, due to the lack of alternative outlets for these types of papers. Therefore, we have instituted a unique process, in which the authors develop a detailed outline of their paper, key tables and figures, and a description of their literature review process. On the basis of this outline, we grant assurances that the contribution hurdle will not be an issue for publication in JAMS, as long as the authors execute the proposed outline as written. Each paper still goes through the normal review process and must meet all publication quality standards, of course. In many cases, an Area Editor takes an active role to help ensure that each paper provides sufficient insights, as required for a high-quality review paper. This process gives the author team confidence to invest effort in the process. An analysis of the marketing journals in the Financial Times (FT 50) journal list for the past five years (2012-2016) shows that JAMS has become the most common outlet for these papers, publishing $31 \%$ of all review papers that appeared in the top six marketing journals.

As a next step in positioning JAMS as a receptive marketing outlet for review papers, we are conducting a Thought Leaders Conference on Generalizations in Marketing: Systematic Reviews and Meta-Analyses, with a corresponding special issue (see www.springer.com/jams). We will continue our process of seeking out review papers as an editorial strategy in areas that could be advanced by the integration and synthesis of extant research. We expect that, ultimately, such efforts will become unnecessary, as authors initiate review papers on topics of their own choosing to submit them to JAMS. In the past two years, JAMS already has increased the number of papers it publishes annually, from just over 40 to around 60 papers per year; this growth has provided "space" for 8-10 review papers per year, reflecting our editorial target.

Consistent with JAMS' overall focus on managerially relevant and strategy-focused topics, all review papers should reflect this emphasis. For example, the domains, theories, and methods reviewed need to have some application to past 
or emerging managerial research. A good rule of thumb is that the substantive domain, theory, or method should attract the attention of readers of JAMS.

The efforts of multiple editors and Area Editors in turn have generated a body of review papers that can serve as useful examples of the different types and approaches that $J A M S$ has published.

\section{Domain-based review papers}

Domain-based review papers review, synthetize, and extend a body of literature in the same substantive domain. For example, in "The Role of Privacy in Marketing" (Martin and Murphy 2017), the authors identify and define various privacy-related constructs that have appeared in recent literature. Then they examine the different theoretical perspectives brought to bear on privacy topics related to consumers and organizations, including ethical and legal perspectives. These foundations lead in to their systematic review of privacyrelated articles over a clearly defined date range, from which they extract key insights from each study. This exercise of synthesizing diverse perspectives allows these authors to describe state-of-the-art knowledge regarding privacy in marketing and identify useful paths for research. Similarly, a new paper by Cleeren et al. (2017), "Marketing Research on Product-Harm Crises: A Review, Managerial Implications, and an Agenda for Future Research," provides a rich systematic review, synthesizes extant research, and points the way forward for scholars who are interested in issues related to defective or dangerous market offerings.

\section{Theory-based review papers}

Theory-based review papers review, synthetize, and extend a body of literature that uses the same underlying theory. For example, Rindfleisch and Heide's (1997) classic review of research in marketing using transaction cost economics has been cited more than 2200 times, with a significant impact on applications of the theory to the discipline in the past 20 years. A recent paper in JAMS with similar intent, which could serve as a helpful model, focuses on "Resource-Based Theory in Marketing" (Kozlenkova et al. 2014). The article dives deeply into a description of the theory and its underlying assumptions, then organizes a systematic review of relevant literature according to various perspectives through which the theory has been applied in marketing. The authors conclude by identifying topical domains in marketing that might benefit from additional applications of the theory (e.g., marketing exchange), as well as related theories that could be integrated meaningfully with insights from the resource-based theory.

\section{Method-based review papers}

Method-based review papers review, synthetize, and extend a body of literature that uses the same underlying method. For example, in "Event Study Methodology in the Marketing Literature: An Overview" (Sorescu et al. 2017), the authors identify published studies in marketing that use an event study methodology. After a brief review of the theoretical foundations of event studies, they describe in detail the key design considerations associated with this method. The article then provides a roadmap for conducting event studies and compares this approach with a stock market returns analysis. The authors finish with a summary of the strengths and weaknesses of the event study method, which in turn suggests three main areas for further research. Similarly, "Discriminant Validity Testing in Marketing: An Analysis, Causes for Concern, and Proposed Remedies" (Voorhies et al. 2016) systematically reviews existing approaches for assessing discriminant validity in marketing contexts, then uses Monte Carlo simulation to determine which tests are most effective.

Our long-term editorial strategy is to make sure JAMS becomes and remains a well-recognized outlet for both metaanalysis and systematic managerial review papers in marketing. Ideally, review papers would come to represent $10 \%$ $20 \%$ of the papers published by the journal.

\section{Process and structure for review papers}

In this section, we review the process and typical structure of a systematic review paper, which lacks any long or established tradition in marketing research. The article by Grewal et al. (2018) provides a summary of effects-focused review papers (i.e., meta-analyses), so we do not discuss them in detail here.

\section{Systematic literature review process}

Some review papers submitted to journals take a "narrative" approach. They discuss current knowledge about a research domain, yet they often are flawed, in that they lack criteria for article inclusion (or, more accurately, article exclusion), fail to discuss the methodology used to evaluate included articles, and avoid critical assessment of the field (Barczak 2017). Such reviews tend to be purely descriptive, with little lasting impact.

In contrast, a systematic literature review aims to "comprehensively locate and synthesize research that bears on a particular question, using organized, transparent, and replicable procedures at each step in the process" (Littell et al. 2008, p. 1). Littell et al. describe six key steps in the systematic review process. The extent to which each step is emphasized varies by paper, but all are important components of the review. 
1. Topic formulation. The author sets out clear objectives for the review and articulates the specific research questions or hypotheses that will be investigated.

2. Study design. The author specifies relevant problems, populations, constructs, and settings of interest. The aim is to define explicit criteria that can be used to assess whether any particular study should be included in or excluded from the review. Furthermore, it is important to develop a protocol in advance that describes the procedures and methods to be used to evaluate published work.

3. Sampling. The aim in this third step is to identify all potentially relevant studies, including both published and unpublished research. To this end, the author must first define the sampling unit to be used in the review (e.g., individual, strategic business unit) and then develop an appropriate sampling plan.

4. Data collection. By retrieving the potentially relevant studies identified in the third step, the author can determine whether each study meets the eligibility requirements set out in the second step. For studies deemed acceptable, the data are extracted from each study and entered into standardized templates. These templates should be based on the protocols established in step 2 .

5. Data analysis. The degree and nature of the analyses used to describe and examine the collected data vary widely by review. Purely descriptive analysis is useful as a starting point but rarely is sufficient on its own. The examination of trends, clusters of ideas, and multivariate relationships among constructs helps flesh out a deeper understanding of the domain. For example, both Hult (2015) and Huber et al. (2014) use bibliometric approaches (e.g., examine citation data using multidimensional scaling and cluster analysis techniques) to identify emerging versus declining themes in the broad field of marketing.

6. Reporting. Three key aspects of this final step are common across systematic reviews. First, the results from the fifth step need to be presented, clearly and compellingly, using narratives, tables, and figures. Second, core results that emerge from the review must be interpreted and discussed by the author. These revelatory insights should reflect a deeper understanding of the topic being investigated, not simply a regurgitation of well-established knowledge. Third, the author needs to describe the implications of these unique insights for both future research and managerial practice.

A new paper by Watson et al. (2017), "Harnessing Difference: A Capability-Based Framework for Stakeholder Engagement in Environmental Innovation," provides a good example of a systematic review, starting with a cohesive conceptual framework that helps establish the boundaries of the review while also identifying core constructs and their relationships. The article then explicitly describes the procedures used to search for potentially relevant papers and clearly sets out criteria for study inclusion or exclusion. Next, a detailed discussion of core elements in the framework weaves published research findings into the exposition. The paper ends with a presentation of key implications and suggestions for the next steps. Similarly, "Marketing Survey Research Best Practices: Evidence and Recommendations from a Review of JAMS Articles" (Hulland et al. 2017) systematically reviews published marketing studies that use survey techniques, describes recent trends, and suggests best practices. In their review, Hulland et al. examine the entire population of survey papers published in JAMS over a ten-year span, relying on an extensive standardized data template to facilitate their subsequent data analysis.

\section{Structure of systematic review papers}

There is no cookie-cutter recipe for the exact structure of a useful systematic review paper; the final structure depends on the authors' insights and intended points of emphasis. However, several key components are likely integral to a paper's ability to contribute.

Depth and rigor Systematic review papers must avoid falling in to two potential "ditches." The first ditch threatens when the paper fails to demonstrate that a systematic approach was used for selecting articles for inclusion and capturing their insights. If a reader gets the impression that the author has cherrypicked only articles that fit some preset notion or failed to be thorough enough, without including articles that make significant contributions to the field, the paper will be consigned to the proverbial side of the road when it comes to the discipline's attention.

Authors that fall into the other ditch present a thorough, complete overview that offers only a mind-numbing recitation, without evident organization, synthesis, or critical evaluation. Although comprehensive, such a paper is more of an index than a useful review. The reviewed articles must be grouped in a meaningful way to guide the reader toward a better understanding of the focal phenomenon and provide a foundation for insights about future research directions. Some scholars organize research by scholarly perspectives (e.g., the psychology of privacy, the economics of privacy; Martin and Murphy 2017); others classify the chosen articles by objective research aspects (e.g., empirical setting, research design, conceptual frameworks; Cleeren et al. 2017). The method of organization chosen must allow the author to capture the complexity of the underlying phenomenon (e.g., including temporal or evolutionary aspects, if relevant).

Replicability Processes for the identification and inclusion of research articles should be described in sufficient detail, such that an interested reader could replicate the procedure. The 
procedures used to analyze chosen articles and extract their empirical findings and/or key takeaways should be described with similar specificity and detail.

Usability We already have noted the potential usefulness of well-done review papers. Some scholars always are new to the field or domain in question, so review papers also need to help them gain foundational knowledge. Key constructs, definitions, assumptions, and theories should be laid out clearly (for which purpose summary tables are extremely helpful). An integrated conceptual model can be useful to organize cited works. Most scholars integrate the knowledge they gain from reading the review paper into their plans for future research, so it is also critical that review papers clearly lay out implications (and specific directions) for research. Ideally, readers will come away from a review article filled with enthusiasm about ways they might contribute to the ongoing development of the field.

Helpful format Because such a large body of research is being synthesized in most review papers, simply reading through the list of included studies can be exhausting for readers. We cannot overstate the importance of tables and figures in review papers, used in conjunction with meaningful headings and subheadings. Vast literature review tables often are essential, but they must be organized in a way that makes their insights digestible to the reader; in some cases, a sequence of more focused tables may be better than a single, comprehensive table.

\section{Conclusion}

In summary, articles that review extant research in a domain (topic, theory, or method) can be incredibly useful to the scientific progress of our field. Whether integrating the insights from extant research through a meta-analysis or synthesizing them through a systematic assessment, the promised benefits are similar. Both formats provide readers with a useful overview of knowledge about the focal phenomenon, as well as insights on key dilemmas and conflicting findings that suggest future research directions. Thus, the editorial team at JAMS encourages scholars to continue to invest the time and effort to construct thoughtful review papers.

\section{References}

Barczak, G. (2017). From the editor: writing a review article. Journal of Product Innovation Management, 34(2), 120-121.

Bem, D. J. (1995). Writing a review article for psychological bulletin. Psychological Bulletin, 118(2), 172-177.

Bettencourt, L. A., \& Houston, M. B. (2001). Assessing the impact of article method type and subject area on citation frequency and reference diversity. Marketing Letters, 12(4), 327-340.

Cleeren, K., Dekimpe, M. G., \& van Heerde, H. J. (2017). Marketing research on product-harm crises: a review, managerial implications. Journal of the Academy of Marketing Science, 45(5), 593-615.

Grewal, D., Puccinelli, N. M., \& Monroe, K. B. (2018). Meta-analysis: error cancels and truth accrues. Journal of the Academy of Marketing Science, 46(1).

Hanssens, D. M. (2018). The value of empirical generalizations in marketing. Journal of the Academy of Marketing Science, 46(1).

Huber, J., Kamakura, W., \& Mela, C. F. (2014). A topical history of JMR. Journal of Marketing Research, 51(1), 84-91.

Hulland, J., Baumgartner, H., \& Smith, K. M. (2017). Marketing survey research best practices: evidence and recommendations from a review of JAMS articles. Journal of the Academy of Marketing Science. https://doi.org/10.1007/s11747-017-0532-y.

Hult, G. T. M. (2015). JAMS 2010 - 2015: literature themes and intellectual structure. Journal of the Academy of Marketing Science, 43(6), 663-669.

Knoll, J., \& Matthes, J. (2017). The effectiveness of celebrity endorsements: a meta-analysis. Journal of the Academy of Marketing Science, 45(1), 55-75.

Kozlenkova, I. V., Samaha, S. A., \& Palmatier, R. W. (2014). Resourcebased theory in marketing. Journal of the Academy of Marketing Science, 42(1), 1-21.

Littell, J. H., Corcoran, J., \& Pillai, V. (2008). Systematic reviews and meta-analysis. New York: Oxford University Press.

Martin, K. D., \& Murphy, P. E. (2017). The role of data privacy in marketing. Journal of the Academy of Marketing Science, 45(2), $135-155$.

Rindfleisch, A., \& Heide, J. B. (1997). Transaction cost analysis: past, present, and future applications. Journal of Marketing, 61(4), 3054.

Sorescu, A., Warren, N. L., \& Ertekin, L. (2017). Event study methodology in the marketing literature: an overview. Journal of the Academy of Marketing Science, 45(2), 186-207.

Verma, V., Sharma, D., \& Sheth, J. (2016). Does relationship marketing matter in online retailing? A meta-analytic approach. Journal of the Academy of Marketing Science, 44(2), 206-217.

Voorhies, C. M., Brady, M. K., Calantone, R., \& Ramirez, E. (2016). Discriminant validity testing in marketing: an analysis, causes for concern, and proposed remedies. Journal of the Academy of Marketing Science, 44(1), 119-134.

Watson, R., Wilson, H. N., Smart, P., \& Macdonald, E. K. (2017). Harnessing difference: a capability-based framework for stakeholder engagement in environmental innovation. Journal of Product Innovation Management. https://doi.org/10.1111/jpim.12394. 